\title{
Registro de ensaios clínicos: a discussão in- ternacional e os posicionamentos possíveis para o Brasil
}

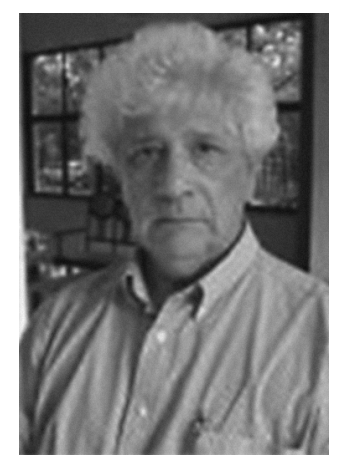

José da Rocha Carvalheiro

Vice-Presidente de Pesquisa e Desenvolvimento Tecnológico da Fundação Oswaldo Cruz, Rio de Janeiro, Brasil jrcarval@fiocruz.br

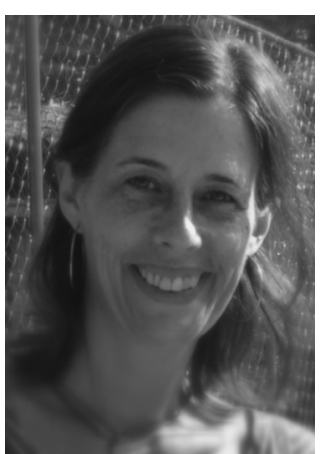

Cristiane

Quental

Escola Nacional de Saúde Pública Sérgio Arouca da Fundação Oswaldo Cruz , Rio de Janeiro, Brasil cquental@fiocruz.br

\section{Resumo}

O objetivo deste texto é apresentar as discussões relativas ao registro de ensaios clínicos em pauta no mundo atualmente e discutir as perspectivas de inserção internacional abertas para o Brasil. Os ensaios clínicos são uma das mais valiosas fontes de evidências sobre a eficácia e a segurança de intervenções em saúde. Estas evidências, entretanto, são divulgadas basicamente através de revistas científicas e sujeitas ao chamado viés de publicação: artigos que apresentem resultados estatisticamente significativos, obtidos a partir de grandes estudos, de boa qualidade, favoráveis à industria, têm maior probabilidade de serem publicados. O registro dos protocolos dos ensaios clínicos em bases de dados públicas, quando do seu início, tem sido apresentado como alternativa de minimizar o problema. O registro de ensaios clínicos tem sido defendido por inúmeras outras razões - éticas, sanitárias, científicas e econômicas. Entretanto, nem os princípios que devem reger estes registros nem sua operacionalização são consensuais, em função dos interesses divergentes de pesquisadores, indústria farmacêutica, governo e sociedade.

\section{Palavras-chave}

Pesquisa clínica, ensaios clínicos, intervenções em saúde, registro, bases de dados

\section{Introdução}

Um ensaio clínico é uma investigação prospectiva, que avalia o efeito de qualquer intervenção deliberada, dirigida a seres humanos, sobre seus parâmetros clínicos (KRLEZA-JÉRIC et al., 2005). Nesse sentido, os ensaios clínicos são uma das mais valiosas fontes de evidências sobre a eficácia e a segurança de intervenções em saúde (SIM, 2006).

Estas evidências, entretanto, são divulgadas basicamente através de revistas científicas - o que acarreta o chamado viés de publicação nas análises que se queira fazer sobre os resultados disponíveis. Os periódicos, por razões óbvias, não podem publicar todos os ensaios realizados no mundo. Precisam optar por apresentar artigos que chamem a atenção e interessem aos seus leitores. Privilegiam, assim, artigos que apresentem resultados estatisticamente significativos, obtidos a partir de grandes estudos, de boa qualidade. Por outro lado, precisam ter artigos do interesse da indústria, que encomendam milhares de exemplares para distribuir aos médicos. Assim, ao submeter um artigo para publicação, 
o pesquisador também seleciona os resultados que vai apresentar, dentre os vários que colheu, num processo de divulgação seletiva (REVEIZ et al., 2006).

No caso do Brasil - e dos demais países latinoamericanos -, a questão da língua de publicação e o fato da maioria de seus periódicos não estarem entre os indexados pelas maiores bases indexadoras, como MEDLINE, reduz ainda mais o acesso internacional aos ensaios aqui realizados (REVEIZ et al., 2006), de grande importância para países em situação equivalente.

O registro dos protocolos dos ensaios clínicos em bases de dados públicas, quando do seu início, tem sido apresentado como alternativa de minimizar o problema. O registro de ensaios clínicos tem sido defendido por inúmeras outras razões: éticas, sanitárias, científicas, tecnológicas e econômicas. O registro seria uma obrigação ética para com quem participou do ensaio, que espera contribuir para o conhecimento científico. Seria também a forma de garantir a pacientes e médicos acesso à informação sobre ensaios que estejam recrutando pacientes. E a pacientes, médicos, investigadores, comitês de ética e patrocinadores acesso a informações sobre ensaios que ocorreram no passado e estão ocorrendo agora: evitando os riscos de exposição desnecessária a intervenções já estudadas, evitando a duplicação de esforços, impulsionando o avanço do conhecimento e a cooperação entre grupos de pesquisa (KRLEZA-JERIC et al., 2005).

CUERVO et al. (2006) destacam, ainda, a importância do registro dos ensaios clínicos para o planejamento da pesquisa, permitindo identificar as lacunas no conhecimento existentes em diferentes áreas, observar tendências no campo dos estudos, além de permitir identificar os especialistas nas diversas áreas. Vale ressaltar que, sendo as atividades de pesquisa e desenvolvimento tecnológico a base da inovação nas indústrias da saúde, estas informações são valiosas tanto para as empresas quanto para os formuladores de políticas de promoção da inovação.

Apesar dos benefícios do registro dos ensaios serem considerados há anos, foi a partir de 2004 que a questão ganhou maior atenção, quando foi movida uma ação pública contra a empresa farmacêutica GlaxoSmithKline pelo Procurador-Geral de Nova York por ocultação de evidências negativas envolvendo seu antidepressivo paroxetina, comercializado como Paxil, nos EUA, e Seroxat, na Grã-Bretanha (KRLEZA-JERIC, 2005). Ainda, segundo DYER (2004), o Procurador dispunha de memorando interno da companhia datado de 1998, dizendo que seria “comercialmente inaceitável admitir que a paroxetina não havia funcionado em crianças e que a companhia deveria gerenciar a disseminação desses dados para minimizar quaisquer impactos negativos".

O caso intensificou o debate sobre a necessidade de implantação de uma base que pudesse registrar todos os ensaios clínicos desde seu início. Mas, embora tenham sido observadas inúmeras iniciativas no sentido de criação de registros e de incentivo ao registro público dos ensaios clínicos ao redor do mundo, nem os princípios que devem reger esses registros nem sua operacionaliza- ção são consensuais, em função dos interesses divergentes de pesquisadores, indústria farmacêutica, governo e sociedade.

O texto apresenta o debate em pauta no mundo atualmente e discute as perspectivas de inserção internacional abertas para o Brasil.

\section{Principais iniciativas no sentido de promover o registro dos ensaios clínicos}

Uma das iniciativas pioneiras partiu do Canadian Institutes of Health Research, que, logo após o episódio com a Glaxo SmithKline, passou a exigir o registro de todos os ensaios por eles financiados (CUERVO et al., 2006) e convocou uma reunião aberta em Ottawa, para a qual convidou os interessados em contribuir na elaboração de um plano para o registro global de ensaios clínicos. A questão foi debatida e se criou o Grupo de Ottawa para levar a discussão adiante, internacionalmente (OTTAWA GROUP, 2007). A Declaração de Ottawa - parte I (KRLEZA-JERIC et al., 2005) foi publicada em vários periódicos, defende o registro de todos os ensaios clínicos aprovados por comitês de ética e autoridades sanitárias, e que estes ganhem um número único de identificação, global. O registro deve ocorrer antes do início do recrutamento dos participantes do estudo, colocando à disposição do público informação sobre o protocolo, sendo esta informação atualizada sempre que forem verificadas mudanças. Defende também o registro dos resultados alcançados, tão logo disponíveis, assim como dos efeitos adversos. Sua divulgação para o público, entretanto, pode esperar a publicação dos resultados, que deve estar relacionada na base. (A parte II da Declaração de Ottawa, que versa sobre os princípios de operacionalização do registro dos ensaios, está aberta no site do grupo (www.ottawagroup.ohri.ca), para consulta pública. A parte III, que trata da abertura dos resultados, está sendo desenvolvida.)

O Grupo defende que o registro deve ser uma exigência legal, mas exorta editores de periódicos a exigirem o número de registro único para a publicação de artigos relacionados a ensaios e os comitês de ética a incentivarem o procedimento.

Essa proposta teve o importante respaldo do International Comittee of Medical Journal Editors (ICMJE), que anunciou, em setembro de 2004, que os periódicos afiliados passariam a aceitar para publicação apenas ensaios registrados em bases públicas que seguissem determinados critérios: acesso do público sem ônus, gerenciadas por organização sem fins lucrativos, dispondo de mecanismo de validação dos dados registrados, e que permitissem buscas eletrônicas. Cada registro deveria incluir um número único de identificação, a intervenção em questão, as comparações estabelecidas, a hipótese em estudo, a definição das variáveis de resultados primárias e secundárias, os critérios de inclusão, o cronograma, o número de sujeitos, as fontes de financiamento, as informações para contato com o investigador principal (DE ANGELIS et al., 2004). Este foi o fator decisivo para 
que os ensaios passassem a ser mais sistematicamente registrados (ZARIN et al., 2005). Essa determinação foi seguida por muitos outros periódicos, inclusive pelos afiliados a Bireme - Centro Latino-Americano e do Caribe de Informação em Ciências da Saúde, da Organização Pan-americana da Saúde - OPAS, através da indexação nas bases LILACS e SciELO, que anunciou que também exigirá, a partir de agosto de 2007 , o registro prévio para publicação de artigos sobre ensaios clínicos (BIREME, 2006).

Ainda em 2004, em novembro, realizou-se, na Cidade do México, uma Cúpula Ministerial voltada para a pesquisa em saúde. A Declaração do México, assinada por Ministros da Saúde e outros participantes de 52 países, reconhece que os resultados de pesquisas de boa qualidade devem ser acessíveis, para informar efetivamente a política de saúde e a tomada de decisão na assistência. Reconhece, ainda, que os resultados de pesquisa devem ser publicados, documentados em registros e arquivos acessíveis internacionalmente, e sintetizados através de revisões sistemáticas da totalidade dos resultados de pesquisa disponíveis, para embasar uma abordagem de saúde baseada em evidências. Insta a Organização Mundial da Saúde - OMS - a facilitar a integração de uma rede internacional de registros de ensaios clínicos, assegurando um único portal de acesso e a identificação dos ensaios sem ambigüidades (MINISTERIAL SUMMIT, 2004).

A OMS aceita a missão, entendendo que, por seu caráter global e neutro, inspira maior confiança do público. Seu papel de autoridade mundial na área da saúde, tendo como função essencial a coordenação e a normatização, a credencia para coordenar a tarefa (SIM, 2006). Na verdade, a OMS já vinha discutindo a questão e, após ampla consulta internacional, lançou, em maio de 2005, a proposta de criação de uma Plataforma Internacional de Registro de Ensaios Clínicos (International Clinical Trials Registry Platform - ICTRP), apresentada a seguir.

\section{A proposta da OMS}

A OMS defende que todos os ensaios sejam registrados. Sua proposta é de liderar o processo de normatização dos registros de ensaios clínicos em nível internacional, para tornar possível a cooperação entre eles, no sentido de formar uma rede, acessível de um único portal, onde cada ensaio receba um número único de identificação que permita acompanhá-lo inequivocamente ao longo de sua existência.

Uma das pedras angulares da proposta é, assim, um conjunto mínimo de informações, que devem ser registradas sobre cada ensaio, enumeradas no Quadro I.

Segundo SIM (2006), existem centenas de bases de registro de ensaios no mundo, que variam em escopo - sendo voltadas para determinada doença, país ou financiador - e finalidades - para acompanhamento administrativo, recrutamento de pacientes, análise científica etc. A OMS reconhece que cada uma tem sua aplicação, não sendo realístico pensar em uma única base que atendesse a todos. Assim, propõe uma estrutura hierárquica de ligação entre elas, descrita na Figura 1.

\section{Quadro 1 - Conjunto de dados para registro no ICTRP}

01. Primary register trial number / Número único de identificação

02. Trial registration date / Data de registro do ensaio

03. Secondary IDs / Identidades secundárias

04. Sources of monetary or material support / Fontes de financiamento e suporte material

05. Primary sponsor /Principal patrocinador

06. Secondary sponsor(s) / Outros patrocinadores

07. Contact for public queries / Contato para dúvidas do público

08. Contact for scientific queries / Contato para dúvidas científicas

09. Public title of the study / Título público do estudo

10. Scientific title / Título científico

11. Countries of recruitment / Países de recrutamento

12. Health condition or problems studied / Problemas de saúde estudados

13. ntervention(s) / Intervenções

14. Key inclusion and exclusion criteria / Critérios de inclusão e exclusão

15. Study type / Tipo de estudo

16. Date of the first enrollment / Data de recrutamento do primeiro voluntário

17. Target sample size / Tamanho da amostra pretendido

18. Recruitment status / Status de recrutamento

19. Primary outcomes / Medidas de resultado primárias

20. Key secondary outcomes / Medidas de resultado secundárias

Fonte: WHO, 2007

\section{Figura 1 - Estrutura da Plataforma ICTRP proposta pela OMS}

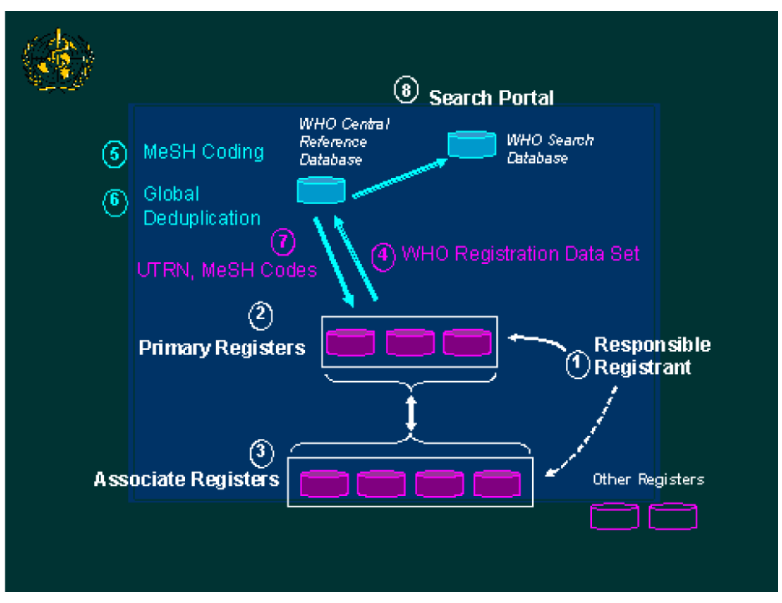

Fonte: SIM, 2006

Nesta hierarquia, Registros Associados enviariam suas informações para Registros Primários, que ficariam responsáveis pelo trabalho de "desduplicação" de registros (identificação de ensaios registrados em mais de uma base) e de tradução para o inglês, quando fosse o caso, já que toda informação tem que ser disponibilizada em inglês. A ICTRP seria um meta-registro articulando a informação registrada nesses Registros Primários. 


\section{Questões em discussão}

São diferentes as opiniões no que tange à natureza e ao alcance que deve ter o registro de ensaios clínicos. A primeira questão polêmica que surge é com relação à própria delimitação de quais ensaios devem ser registrados. Enquanto a proposta do Grupo de Ottawa e a da OMS é de que todos os ensaios devem ser registrados, a indústria pede que os ensaios de caráter exploratório, realizados nas fases iniciais da pesquisa, sejam dispensados do registro, uma vez que visam meramente gerar hipóteses de trabalho. Afetariam diretamente sua competitividade sem contribuir efetivamente como evidência médica, não sendo relevantes para informar a prática clínica (KRLEZA-JERIC, 2005). O ICMJE seguiu essa segunda racionalidade, definido o ensaio a ser registrado como um projeto de pesquisa que, prospectivamente, sujeita pessoas a intervenções médicas para estudar relações de causa e efeito com relação a resultados de saúde. "Estudos desenhados para outros propósitos, como para estudar farmacocinética ou toxicidade (como ensaios de fase I) estão dispensados" (DE ANGELIS et al., 2004). A esses argumentos contrapõem-se, entretanto, os recentes eventos que levaram à morte de uma pessoa em um ensaio de fase I, em Londres, como ilustrando a necessidade de registro de todos os ensaios (HEALTH CANADA, 2006).

A indústria pede também que cinco dos 20 itens integrantes do conjunto básico de informações proposto pela OMS tenham sua divulgação retardada: título científico oficial do estudo, intervenções, resultados primários, principais resultados secundários e tamanho da amostra - itens mais sensíveis para a competição. O Grupo de Ottawa é veemente contra essa proposta, enfatizando que a proposta da OMS já deixa de fora itens considerados pela Declaração de Ottawa como cruciais, e que aqueles já estariam todos na base de dados do ClinicalTrials.gov, ao qual a indústria já estaria acostumada (KRLEZAJERIC, 2005). Argumenta que, considerando-se que um dos campos é relativo ao número único e nove são administrativos, sobraria apenas um conjunto mínimo de dez variáveis que descrevem o ensaio e que sem as cinco principais, a descrição ficaria absolutamente genérica e sem utilidade. Se ainda poderia servir para incluir pacientes em estudos, um tal registro não serviria aos objetivos éticos e científicos (KRLEZA-JERIC, 2005).

A proposta da OMS é de só dar o número único de registro para aqueles que preencherem os 20 itens (embora possa considerar a revelação de alguns deles em momento posterior). A experiência do ClinicalTrials.gov, o maior registro do mundo com cerca de 39 mil ensaios registrados (mantido pela National Library of Medicine - National Institutes of Health/EUA), descrita em ZARIN et al. (2005), mostra que a qualidade dessas informações pode ser bastante ruim, mesmo considerando-se que o ClinicalTrials.gov aceita as informações como são registradas: no período de maio a outubro de 2005, o campo relativo à principal variável de medida do ensaio foi preenchido em apenas $76 \%$ dos ensaios patrocinados pela indústria, e a qualidade dos dados deixa a desejar, como mostra o Quadro 2.

\section{Quadro 2 - Medidas de resultado primárias no ClinicalTrials.gov (mai-out 2005)}

Em $76 \%$ dos ensaios patrocinados pela indústria este campo estava preenchido. Porém:

- em 17\% o campo estava preenchido de forma vaga;

- em 19\% foi apresentando o domínio, sem medida específica;

- em 23\% foi apresentada a medida, porém sem o horizonte de tempo;

- em $10 \%$ foi apresentado o horizonte de tempo, porém sem a medida;

- em 31\% foram apresentados a medida específica e o horizonte de tempo.

Fonte: Zarin et al. (2005)

Mas há discordâncias também quanto ao modelo do sistema. A proposta da OMS é de uma estrutura hierárquica, onde o registro dos ensaios seria feito em registros secundários, nacionais ou regionais, que seriam consolidados por registros primários. O ClinicalTrials.gov propõe o inverso: que os ensaios sejam registrados primeiro nas bases primárias, que distribuiriam os registros de interesse para as secundárias. Acham que a consolidação é trabalhosa e não garantiria a qualidade dos dados. Por outro lado, o Current Controlled Trials (www.controlledtrials.com), o segundo maior registro do mundo, criado na Inglaterra por pesquisadores dedicados principalmente à revisão sistemática, organizados pela editora Current Science Group, com mais de 5 mil ensaios repertoriados, é um meta-registro, organizado em estrutura semelhante à proposta pela OMS. Agrega vários outros registros e possui um esquema de numeração única de ensaios, o International Standard Randomized Controlled Trial Number (ISRCTN).

A adesão internacional à proposta é fundamental na ausência de mecanismos que obriguem o registro. Embora exista alguma legislação em vigor e inúmeros projetos tramitando no mundo nesse sentido, o maior incentivo ao registro ainda é a exigência dos periódicos para a publicação de artigos. O momento é de negociação e busca de apoios institucionais, pois o registro é voluntário.

\begin{abstract}
"It should be stressed, however, that any limitiation on the availability of information would result in a limitation on the efficiency of Clinical Trials Registers, and thus would represent a possible functional and ethical breach". (BOISSEL et al, 1993)
\end{abstract}

\section{Movimentos nas Américas/Alternativas para a posição brasileira}

A proposta da OMS, defendida pela OPAS, tem obtido apoio nas Américas. Foi criado o LATINREC - The Latin American Ongoing Clinical Trial Register, desenvolvido pela braço colombiano da Colaboração Cochrane Iberoamericana - organização independente de informação em saúde que congrega 12 centros colaborativos em dez 
países latino-americanos (Argentina, Chile, Colômbia, Costa Rica, Cuba, Equador, Guatemala, México, Peru e Venezuela), coordenados pelo centro de Barcelona. Este registro está para iniciar suas operações, seguindo os requisitos da ICTRP. Ao lado do ClinicalTrials.gov, seriam dois os registros da rede nas Américas.

O Canadá também aderiu à proposta, mas discute como melhor implementá-la: criar um registro nacional ou se associar a um registro que atenda às condições da ICTRP, como o ClinicalTrials.gov. Está tendendo a uma opção de associação customizada, onde, além das informações do registro, pede outras - no caso, o termo de consentimento do voluntário (HEALTH CANADA, 2006).

A importância da participação brasileira no esforço global é clara, contribuindo para incentivar o registro no país, dar maior visibilidade aos ensaios aqui realizados e contribuir para uma maior qualidade dos dados disponibilizados. Reforça, também, o sistema de revisão ética e sanitária e os princípios que os regem, além de servir para subsidiar políticas de saúde, científicas e tecnológicas, nacionais ou institucionais.

As discussões sobre a proposta da OMS, inicialmente restritas ao âmbito das autoridades responsáveis, como o Departamento de Ciência e Tecnologia - Decit, da Secretaria de Ciência, Tecnologia e Insumos Estratégicos - SCTIE, do Ministério da Saúde (que encampou a proposta), a Agência Nacional de Vigilância Sanitária - Anvisa - e o Conselho Nacional de Ética em Pesquisa Conep, ganharam fóruns mais amplos no VIII Congresso Brasileiro em Saúde Coletiva e XI Congresso Internacional em Saúde Pública, realizado no Rio de Janeiro, em agosto de 2006.

As atividades promovidas por OPAS, Decit e Fiocruz contaram com a presença de representantes do governo, de editores, da indústria e dos pacientes, de registros estrangeiros (Latinrec e registro da África do Sul) e nacionais (ICICT/Fiocruz), de pesquisadores, enfim, uma gama bastante ampla de interessados, que culminaram na decisão de criação de uma base nacional.

Colocam-se para nós as mesmas questões que enfrentam os canadenses: como melhor implementar a proposta? Vale a pena criar um Registro Nacional? O Quadro 3 mostra algumas estatísticas relativas aos ensaios registrados no ClinicalTrails. gov em março de 2007. Segundo o Quadro, haveria 511 estudos registrados na base que listavam o Brasil como uma das localidades de realização - cerca de 1,5\% dos ensaios da base. Entretanto, apenas 82 dentre estes listavam exclusivamente o Brasil. Seriam pelo menos esses os ensaios que estariam registrados em nossa base. Este número, entretanto, deve aumentar, uma vez que no último Congresso Internacional de Saúde Pública foi anunciada a decisão dos periódicos latino-americanos de, dentro de um ano, também só aceitar para publicação ensaios registrados, como mencionado anteriormente.

Seria uma melhor opção - como acredita o Canadá - associar-se a um registro que atenda às condições da ICTRP? O interesse de um registro como o ClinicalTrials,

\section{Quadro 3 - Principais países em número de registros de ensaios das Américas}

\begin{tabular}{|c|c|c|c|}
\hline País & Total de ensaios & Em um só país & Em vários países \\
\hline EUA & 23187 & 19995 & 3192 \\
\hline Canadá & 3299 & 1312 & 1987 \\
\hline Alemanha & 2339 & 916 & 1423 \\
\hline França & 2121 & 914 & 1207 \\
\hline Inglaterra & 1867 & 632 & 1235 \\
\hline Brasil & 511 & 82 & 429 \\
\hline México & 458 & 46 & 412 \\
\hline Argentina & 425 & 19 & 406 \\
\hline Chile & 209 & 12 & 197 \\
\hline \multicolumn{4}{|c|}{ Fonte: ClinicalTrials.gov, acessado em 08/03/2007. }
\end{tabular}

por exemplo, numa parceria com o Brasil, seria no incentivo ao registro e na validação de seus dados relativos aos ensaios realizados no território nacional e na divulgação em língua nacional. É esse, inclusive, o objetivo da OMS, ao propor uma estrutura descentralizada - a proximidade do registro dos ensaios realizados, facilita essas tarefas. Para o Brasil, o interesse estaria no estabelecimento de um registro a custo reduzido, pois não teria que financiar a infra-estrutura técnica necessária.

Uma série de outras decisões precisa ser tomada para a implantação de um Registro Nacional, e uma delas é a política de registro do País. O registro pode ser considerado voluntário, ou ter alguma forma de obrigatoriedade. Esta obrigatoriedade não pode afugentar os estudos da indústria. Como um caminho intermediário pode novamente ser citada a discussão canadense (HEALTH CANADA, 2006), que sugere que o governo exija inicialmente apenas que os ensaios que financia sejam registrados, mas que faça gestões para que outras instituições canadenses sigam seu exemplo.

\section{Considerações finais}

Qualquer que seja a opção do Brasil, o importante é que os dados registrados sejam usados, principalmente, pelo Ministério da Saúde, que os financiará.

O principal benefício será conhecer o que acontece no Brasil em termos de ensaios clínicos: Quais são os projetos desenvolvidos? Patrocinados por quem? Executados por quem? Sua utilização mais direta seria no subsídio à política científica e tecnológica e de inovação no complexo industrial da saúde, quer incentivando atividades de $P \& D$, onde as necessidades de saúde pública não estejam sendo atendidas pela indústria, quer regulando o processo, incentivando a geração de conhecimentos para subsidiar a incorporação de novos produtos e novas tecnologias ao sistema de saúde.

Mas os benefícios do registro dos ensaios clínicos ultrapassam a ação governamental no que diz respeito à inovação, contribuindo para a geração de novos produtos: acrescentando novos conhecimentos ao estoque do qual 
bebe a P\&D farmacêutica e da maioria dos insumos para a saúde; sinalizando o que está sendo estudado e onde há lacunas, onde as chances para a inovação são mais favoráveis, pela menor competição a ser enfrentada. É, ao mesmo tempo, uma vitrine para pesquisadores/centros de pesquisa nacionais, propiciando maior interação e inserção internacional, além de facilitar o acesso das empresas aos recursos de que necessita; e uma vitrine para as empresas, atraindo parcerias e facilitando a busca de parcerias.

Espera-se ter contribuído para divulgar a discussão e incentivar o debate a respeito do registro dos ensaios clínicos, não só para subsidiar os movimentos do governo, como para incentivar o registro dos ensaios realizados no Brasil.

\section{Referências bibliográficas}

BIREME. Clinical trials should be registered before publication in LILACS and SciELO journals. Newsletter Biblioteca Virtual em Saúde. Disponível em: www.who. int Acesso em 26 de setembro de 2006.

BOISSEL, J.P. et al. International collaborative group on clinical trail registries: technical report. Clinical Trials and Meta-Analysis, n.28, p.255-266, 1993.

CLINICALTRIALS.GOV. disponível em www.clinicaltrials.gov. Acesso em 08 mar. 2007.

CUERVO, L.G.; VALDÉS, A.; CLARK, M.L. El registro internacional de ensayos clínicos. Editorial. Revista Panamericana de Salud Pública, ano 85, v.19, n.6, jun 2006.

De ANGELIS, C.D. et al. Clinical trial registration: a satetment from the International Committee of Medical Journal Editors. Journal of the American Medical Association n.292, p.1363-1364, set. 2004.

DYER, O. GlaxoSmithKline faces US lawsuit over concealment of trial results. BMJ, n.328, p.1395, jun 2004.
HEALTH CANADA - External working group on the registration and disclosure of clinical trial information. Final report: options for improving public access to information on clinical trials of heath products in $\mathrm{Ca}$ nada. Dec. 2006. Disponível em: http://hc-sc.gc.ca/dhpmps/prodpharma/activit/sci-consult/ewg-ct Acesso em: 02 abr. 2007.

KRLEZA-JÉRIC, K. et al.. Principles for international registration of protocol information and results from human trials of health realted interventions: Ottawa statement (part I). BMJ, v.330 n.7497, p. 956-8, 2005.

KRLEZA-JÉRIC, K. Clinical trial registration: the differing views of industry, the WHO, and the Ottawa Group. PloS Medicine, v.2, n.11, 1093, p.97, nov 2005.

MINISTERIAL SUMMIT ON HEALTH RESEARCH. Knowledge for better health: strenghthening health systems. Mexico City, nov 2004. Disponível em: www. ottawagroup.ohri.ca Acesso em: abr. 2007.

OTTAWA GROUP. Ottawa statement on clinical registration. www.ottawagroup.ohri.ca Acesso em: abr. 2007.

REVEIZ, L. et al. The latin american ongoing clinical trial register (LATINREC). Revista Panamericana de Salud Pública, v. 19, n.6, jun. 2006.

SIM, I.WHO International Clinical Trials Registry Platform. Congresso Brasileiro de Saúde Coletiva, 8. e Congresso Internacional de Saúde Pública, 11. Anais... Rio de Janeiro, 2006.

WORLD HEALTH ORGANIZATION. International Clinical Trials Registry Platform. Disponível em: www. who.int Acesso em 26 mar. 2006.

ZARIN, D. A.; TSE, T.; IDE N. Trial registration at clinical trails.gov between May and October 2005. The New England Journal of Medicine, n. 353, p.27792787, 2005 . 


\section{Sobre os autores}

\section{José da Rocha Carvalheiro}

Possui graduação pela Faculdade de Medicina da Universidade de São Paulo (1961), é professor da Faculdade de Medicina de Ribeirão Preto da USP desde 1963 onde obteve os títulos de Doutor em Parasitologia (1970), Livre Docente (1975), Professor Adjunto (1980) e Professor Titular (1987) no Departamento de Medicina Social. Atualmente, aposentado da USP, é Vice Presidente de Pesquisa e Desenvolvimento Tecnológico da Fundação Oswaldo Cruz onde coordena o Projeto Inovação em Saúde. É membro e atual Presidente da Associação Brasileira de Pós Graduação em Saúde Coletiva (Abrasco), Editor Científico da Revista Brasileira de epidemiologia , Membro do Centro Brasileiro de Estudos de Saúde (Cebes) e do Conselho Editorial da Revista Brasileira de Vigilância Sanitária. Tem experiência na área de Saúde Coletiva, com ênfase em Epidemiologia, atuando principalmente nos seguintes temas: epidemiologia; aids; ciência, tecnologia e inovação em saúde; políticas de desenvolvimento de vacinas e imunobiológicos. Recentemente foi nomeado Vice Presidente de Pesquisa e Desenvolvimento da Fundação Oswaldo Cruz.

\section{Cristiane Quental}

Economista, formada pela Faculdade de Economia e Administração da Universidade Federal do Rio de Janeiro (1982), com mestrado e doutorado em Administração pelo Instituto de Pós-Graduação em Administração (COPPEAD) da mesma Universidade (1989 e 1995). Tem Pós-doutorado no Departamento de Política Científica e Tecnológica do Instituto de Geociências da Universidade Estadual de Campinas (2006). É Pesquisadora da Escola Nacional de Saúde Pública Sérgio Arouca (ENSP) da Fundação Oswaldo Cruz, com interesse nos temas: Política e Gestão de Ciência, Tecnologia e Inovação em Saúde; Sistema Nacional de Inovação em Saúde; Pesquisa e Desenvolvimento em Saúde; Gestão de Instituições Públicas de Pesquisa e Complexo Industrial da Saúde. Coordena o Mestrado Profissional em Gestão de Ciência e Tecnologia em Saúde, oferecido pela ENSP a gestores de organizações federais de ciência e tecnologia em saúde e assessora o Projeto Inovação em Saúde, da Presidência da Fiocruz, instância que está participando do processo de implantação de um Registro de Ensaios Clínicos no Brasil. 